\title{
Spermine Is a Potent Plant Defense Activator Against Gray Mold Disease on Solanum lycopersicum, Phaseolus vulgaris, and Arabidopsis thaliana
}

\author{
Hamed S. Seifi, ${ }^{1}$ Adel Zarei, ${ }^{1}$ Tom Hsiang, ${ }^{2}$ and Barry J. Shelp ${ }^{1, \dagger}$ \\ ${ }^{1}$ Department of Plant Agriculture, University of Guelph, Guelph, Ontario N1G 2W1, Canada \\ ${ }^{2}$ School of Environmental Sciences, University of Guelph, Guelph, Ontario N1G 2W1, Canada \\ Accepted for publication 12 April 2019.
}

\begin{abstract}
Polyamines (PAs) are ubiquitous aliphatic amines that play important roles in growth, development, and environmental stress responses in plants. In this study, we report that exogenous application of spermine $(\mathrm{Spm})$ is effective in the induction of resistance to gray mold disease, which is caused by the necrotrophic fungal pathogen Botrytis cinerea, on tomato (Solanum lycopersicum), bean (Phaseolus vulgaris), and Arabidopsis thaliana. High throughput transcriptome analysis revealed a priming role for the Spm molecule in the genus Arabidopsis, resulting in strong upregulation of several important defense-associated genes, particularly those involved in systemic-acquired resistance. Microscopic analysis confirmed that Spm application potentiates endogenous defense responses in tomato leaves through the generation of reactive
\end{abstract}

ABSTRACT

Botrytis cinerea, the causal agent of the gray mold disease, is the most economically important necrotrophic fungal plant pathogen in the world (Dean et al. 2012). Gray mold causes significant losses to various crops, including those growing under greenhouse or vineyard conditions and during postharvest storage, which is estimated at $\sim \$ 10$ to $\$ 100$ billion per year (Zhao et al. 2009). Although conventional fungicides against the gray mold disease are available, there are situations where they do not offer complete control, and negative health and environmental consequences have been ascribed to some of them. Furthermore, this fungal pathogen possesses a high level of genetic plasticity and has developed resistance against a variety of pre- and postharvest fungicides (Jurick et al. 2017; Williamson et al. 2007). Therefore, the development of novel resistance-enhancing and ecofriendly controls for such an economically impactful disease is important for the production of field and horticultural crops as well as organic production in particular.

In response to phytopathogens, plants have evolved a sophisticated innate immune system consisting of both constitutive and inducible defense mechanisms. Inducible defense mechanisms include the generation of reactive oxygen species (ROS) (e.g., $\mathrm{H}_{2} \mathrm{O}_{2}$ ), the hypersensitive response (HR), the fortification of cell walls, and de novo synthesis of antimicrobial compounds, such as pathogenesis-related (PR) proteins and phytoalexins (Heath 2000; Jones and Dangl 2006). These defenses are normally quiescent in healthy plants; however, they are rapidly and strongly induced in response to a pathogenic challenge after timely perception of

†Corresponding author: B. J. Shelp; bshelp@uoguelph.ca

Funding: This research was supported by Mitacs Canada, Natural Sciences and Engineering Research Council of Canada I2I Program, and NutriAg Ltd.

*The $\boldsymbol{e}$-Xtra logo stands for "electronic extra" and indicates that three supplementary figures and four supplementary tables are published online.

The author(s) declare no conflict of interest.

(c) 2019 The American Phytopathological Society oxygen species and the hypersensitive response, which effectively contained B. cinerea growth within the inoculated area. Moreover, coapplication of Spm and salicylic acid resulted in a synergistic effect against the pathogen, leading to higher levels of resistance than those induced by separate applications of the two compounds. The Spm plus salicylic acid treatment also reduced infection in systemic nontreated leaves of tomato plants. Our findings suggest that Spm, particularly when applied in combination with salicylic acid, functions as a potent plant defense activator that leads to effective local and systemic resistance against $B$. cinerea.

Keywords: biochemistry, cell biology

specific stress signals (Meldau et al. 2012). Some nonpathogenic stimuli, such as root colonization by specific rhizobacteria and contact with certain synthetic or natural compounds known as plant defense activators (PDAs), also induce defense mechanisms in plants, a phenomenon commonly referred to as induced resistance (Walters et al. 2005). A well-characterized type of induced resistance is systemic-acquired resistance (SAR), which results in durable protection against a broad spectrum of pathogens (Conrath et al. 2006). PDAs elicit SAR in plants by boosting key defense-associated pathways, particularly the salicylic acid (SA) pathway (Gaffney et al. 1993), which provokes a "priming" state in the host, thereby leading to stronger and quicker responses to future pathogenic attacks (Aranega-Bou et al. 2014).

Polyamines (PAs) are ubiquitous aliphatic amines that serve various physiological and developmental roles in plants (Galston and Sawhney 1990). The biosynthesis and degradation of PAs are very responsive to different abiotic and biotic stimuli, suggesting that they function in plant-stress interactions (Alcázar et al. 2006; Walters 2003b). Furthermore, the major plant PAs, such as putrescine (Put), spermidine (Spd), and spermine (Spm), as well as their catabolic product diaminopropane (DAP) are known to modulate plant defense responses to phytopathogens (Cona et al. 2006; Jiménez-Bremont et al. 2014; Minocha et al. 2014; Walters $2003 \mathrm{~b}$ ). A specific signaling role for Spm in the induction of plant defense responses has been proposed (Mitsuya et al. 2009). Spm is known to accumulate in the intercellular spaces of tobacco mosaic virus-infected plants (Yamakawa et al. 1998), and Spm application/ accumulation induces mitochondrial membrane dysfunction and $\mathrm{H}_{2} \mathrm{O}_{2}$ generation; expression/accumulation of defense-related proteins, such as PR and protein kinases; and resistance of plants to tobacco mosaic virus, cucumber mosaic virus, Pseudomonas syringae, or Hyaloperonospora arabidopsidis (Marco et al. 2014; Mitsuya et al. 2009; Takahashi et al. 2003, 2004; Yamakawa et al. 1998). Here, we demonstrated that, (i) compared with other PAs, exogenous application of Spm specifically induces strong resistance to B. cinerea in tomato, bean, and Arabidopsis plants; (ii) application of Spm primes the defense response in Arabidopsis 
through induction of SAR and HR; and (iii) coapplication of Spm and SA results in a synergistic interaction with respect to disease suppression in the tomato- $B$. cinerea pathosystem. These findings provide novel insights into the effectiveness of Spm as a potent PDA against the most economically important necrotrophic phytopathogen, $B$. cinerea. Our study provides valuable information that could be used in the development of novel ecofriendly methods for plant disease management in sustainable crop production systems.

\section{MATERIALS AND METHODS}

Plant and fungal growth conditions. Tomato (Solanum lycopersicum L. cultivar Moneymaker), Arabidopsis (Arabidopsis thaliana L. Heyhn ecotype Columbia), and bean (Phaseolus vulgaris L. cultivar Yellow Bush Bean) plants were grown on Sunshine mix LC1 (Sungro Horticulture Inc.) potting soil in a naturally lighted greenhouse supplemented with high-pressure sodium lighting ( $\sim 65 \mathrm{mmol} \mathrm{m}^{-2} \mathrm{~s}^{-1}$ at pot level) and provided with 16-h light at $25^{\circ} \mathrm{C}$ and 8 -h dark at $20^{\circ} \mathrm{C}$. Plants were fertilized with $200 \mathrm{ml}$ of N-P-K (20-7-20) solution once weekly.

$B$. cinerea isolate 1305 was grown on $2 \%$ potato dextrose agar at 20 to $22^{\circ} \mathrm{C}$ in the dark. Plates were completely covered in mycelium after 1 week, and then, they were shifted to a 12-h light/12-h dark regime for 1 week to stimulate sporulation. A highly concentrated stock of $B$. cinerea conidial suspension was freshly prepared by washing spores from fully sporulated plates with sterile water. The final $B$. cinerea inoculation suspension was composed of one quarterstrength potato dextrose broth (PDB) containing $5 \times 10^{5}$ and $10^{5}$ spores $\mathrm{ml}^{-1}$ in leaf disk and whole/detached leaf infection trials, respectively.

Inoculation method and infection trials. Infection trials with floating leaf disks were conducted essentially following Asselbergh et al. (2007). Briefly, leaf disks ( $1 \mathrm{~cm}$ in diameter) were cut using a cork borer from the fifth and sixth leaves of 5-week-old tomato plants, the first and second leaves of 2-week-old bean plants, and rosette leaves of 4-week-old Arabidopsis plants, and they were individually floated overnight in a 24-well plate on $1 \mathrm{ml}$ of aqueous solutions of the desired compounds (i.e., Spm, Spd, Put, and DAP at specific concentrations), whereas control leaf disks were floated on sterile deionized water. After $16 \mathrm{~h}$, a 10- $\mu \mathrm{l}$ droplet of the inoculation suspension was placed on the adaxial surface of each leaf disk, and the plates were incubated at $\sim 22^{\circ} \mathrm{C}$ under dark conditions. Lesion diameters were measured at 3 to 5 days postinoculation (dpi) using an electronic caliper.

Infection trials were also conducted with whole detached fifth and sixth leaves of 5-week-old tomato plants (Seifi et al. 2013a). Briefly, the abaxial surface of the leaves of intact tomato plants was sprayed until runoff with the desired mixture of compounds, including $0.05 \%$ nonylphenol ethoxylate as an adjuvant, and the leaves were placed on four layers of moistened paper towels in a plastic tray sealed with plastic wrap. After $16 \mathrm{~h}$, droplets of the inoculum suspension $(2 \times 10-\mu \mathrm{l}$ droplets per leaflet and 10 droplets per leaf in total) were placed on the adaxial surface of each leaf. For systemicity trials, the first, second, and third leaves of intact plants were sprayed, and after $16 \mathrm{~h}$, the fifth and sixth nonsprayed leaves were detached before inoculation as described above. Lesion diameters were measured at $3 \mathrm{dpi}$ as described above.

Visualization of defense responses. Inoculated leaf disks were sampled at several time points (hours postinoculation [hpi]), and they were cleared and fixed using $100 \%$ ethanol. Then, the leaf disks were mounted in $50 \%$ glycerol for additional microscopic analyses. The defense response was evaluated macroscopically and microscopically using several staining techniques. Cell wall fortifications were monitored by incubating the leaf disks in $0.01 \%$ safranin-O in $50 \%$ ethanol for 3 min followed by several washes in deionized water (Seifi et al. 2013a). Likewise, fungal structures were stained with $0.1 \%$ trypan blue in $10 \%$ acetic acid for $30 \mathrm{~s}$ and then, thoroughly washed (Seifi et al. 2013a). $\mathrm{H}_{2} \mathrm{O}_{2}$ generation/accumulation was visualized as a brown reddish polymer sedimented in the leaf tissue by adding $2 \mathrm{mg} \mathrm{ml}^{-1}$ of $3,3^{\prime}$ diaminobenzidine (DAB) to a final concentration of $1 \mathrm{mg} \mathrm{ml}^{-1} 3 \mathrm{~h}$ before the sampling time (Asselbergh et al. 2007).

Statistical analysis. A linear mixed effects model was used to test for differences among the response to exogenous application of the various compounds studied, wherein "treatment" and "block" were assigned as "fixed" and "random" effects, respectively. The infection trials with tomato and bush bean were conducted with a randomized complete block design containing three or four blocks (i.e., plates or trays), wherein each treatment and/or sampling time in the block consisted of leaf samples derived from at least three different plants. Assumptions of normality for the data were confirmed using a Shapiro-Wilk test, and mean comparisons were conducted using a one-way analysis of variance in combination with Tukey's honest significant difference test $(P \leq 0.05)$. All statistical analyses were performed using $\mathrm{R}$ version 3.4.4. (R Core Team).

RNA extraction, sequencing, and data analysis. Arabidopsis$B$. cinerea infection assays were conducted as described above with two modifications. Detached rosette leaves were incubated on $10 \mathrm{mM} \mathrm{Spm}$ for $16 \mathrm{~h}$ and then, inoculated with multiple $10-\mathrm{ml}$ droplets to cover maximal surface area of the leaves with $B$. cinerea conidial suspension. Also, leaves were mock inoculated with one quarter-strength PDB without spores. Leaves were sampled at $16 \mathrm{hpi}$, flash frozen in liquid nitrogen, and stored at $-80^{\circ} \mathrm{C}$. In total, four treatments were prepared: (i) $\mathrm{Spm}$ treated and B. cinerea inoculated designated as SI, (ii) Spm treated and mock inoculated designated as SM, (iii) water treated and B. cinerea inoculated designated as WI, and (iv) water treated and mock inoculated designated as WM. For each treatment, one leaf each was collected from nine different plants and eventually pooled into three replicates (i.e., $n=$ 3; each replicate contained one leaf from three different plants at the time of sampling). Also, extra leaves (from three different plants) were similarly inoculated and incubated for at least 3 dpi to monitor the progress of the infection.

Leaf samples were ground with a chilled mortar and pestle, and total RNA was extracted using the Bio Basic Rapid Plant RNA Isolation Kit (catalog number PT4191; Bio Basic Inc.) following the manufacturer's instructions. RNA quantity and quality were assessed using the Agilent 2100 BioAnalyser system (Agilent Technologies) at the Genomics Facility of the University of Guelph. High-quality RNA samples (RNA integrity number 26.5 ) were sequenced at the Genome Quebec Innovation Center at McGill University in Montreal, Canada.

All RNA-seq data were analyzed using the Galaxy platform (accessible at https://usegalaxy.org/). Briefly, quality control of data was performed using FastQC (Andrews 2010) (accessible at http:// www.bioinformatics.babraham.ac.uk/projects/fastqc). Quality and adaptor trimmings were done using the Trimmomatic tool (Bolger et al. 2014). The Tuxedo-Suite pipeline (i.e., Tophat2, Cufflinks, Cuffmerge, and Cuffdiff) (Trapnell et al. 2012) was used for read alignment and mapping against $A$. thaliana TAIR10 Col-0 reference genome, transcriptome assembly, merging of assembly data, and differential gene expression analysis. The R package CummeRbund was used for visualization of the data generated. Significantly differentially expressed genes were identified as those with $P$ value $\leq 0.01$ and $\log _{2}$ fold change $\geq 2$. This output was used for Venn diagram generation using jvenn (Bardou et al. 2014) (accessible at http:// jvenn.toulouse.inra.fr/app/example.html) and gene ontology enrichment (GOE) analysis using ClueGO (Bindea et al. 2009) (accessible at http://apps.cytoscape.org/apps/cluego) and AgriGO (Tian et al. 2017).

\section{RESULTS}

Application of Spm strongly suppressed B. cinerea infection of tomato and bean leaf disks. Exogenous application of $1,2.5$, or $5 \mathrm{mM}$ Spm was more effective than the corresponding level of Spd, Put, or DAP in suppressing B. cinerea infection of tomato leaf disks by $3 \mathrm{dpi}$; notably, there was $75 \%$ disease 
reduction with $5 \mathrm{mM}$ Spm compared with the inoculated control (Fig. 1A). The disease suppression level was not affected by lowered $\mathrm{pH}$ of the incubating solution, because similar results were obtained with the addition of $0.1 \mathrm{M} 2$-( $N$-morpholino)-ethane-sulfonate buffer $(\mathrm{pH}$ 6) to the incubation solution (data not shown). Macroscopic analysis of the samples treated with $5 \mathrm{mM} \mathrm{Spm} \mathrm{revealed} \mathrm{that} \mathrm{the} \mathrm{pathogen} \mathrm{was}$ restricted to the challenged area (i.e., the area located beneath the inoculation droplet) as well as the presence of a presumed HR ring around that area (Fig. 1B). Histochemical analysis of fungal structures and cell wall modifications revealed that the HR ring, containing B. cinerea mycelium, was located in the mesophyll (Fig. $1 C)$. Similarly, $1 \mathrm{mM}$ Spm reduced infection by $\sim 85 \%$ in bean leaf disks (Supplementary Fig. S1).

Application of Spm strongly suppressed $B$. cinerea infection of Arabidopsis leaves and upregulated genes controlling the immune response, particularly SAR. Exogenous application of $\mathrm{Spm}$ onto rosette leaves of the genus Arabidopsis suppressed B. cinerea infection of Arabidopsis leaves in a dose-dependent manner, with the $10 \mathrm{mM}$ level resulting, on average, in $88 \%$ disease suppression (Fig. 2A and B). Microscopic analysis showed that the $B$. cinerea mycelium grew normally on Spm-treated leaves, but no lesions were induced, whereas watertreated samples showed severe lesion development (Fig. 2C). Visualization of the defense-associated $\mathrm{H}_{2} \mathrm{O}_{2}$ generation with DAB showed an early accumulation of ROS at 16 hpi in Spm-treated and
B. cinerea-inoculated leaf samples, which was not visible at 24 or $48 \mathrm{~h}$ (Fig. 2D). Subsequently, the effect of $10 \mathrm{mMSpm}$ treatment on global patterns of gene expression in Arabidopsis rosette leaves at $16 \mathrm{hpi}$ was evaluated through RNA-seq. A summary of read alignments and mapping coverage is provided in Supplementary Table S1. Supplementary Figures S2 and S3 summarize the density plot and principal component analyses of the transcripts detected across different treatment groups. The overall pattern of the two plots indicates that the Spm treatment induced a distinctive and strong transcriptional reconfiguration in the genus Arabidopsis. The number of shared and specifically upregulated genes (cuff offs: $P \leq 0.01, \log _{2}$ fold change $\geq 2$ ) in the WI, SM, and SI treatment groups versus the control treatment (WM) are shown in Figure 3A (a complete list of these genes is provided in Supplementary Table S2). A set of 2,599 genes that were exclusively upregulated in response to Spm but were not overlapping

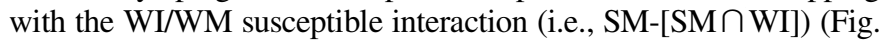
$3 \mathrm{~A}$, dashed area) was selected for the downstream GOE analysis. These differentially expressed genes (DEGs) highlighted significantly activated molecular functions related to defense signaling and response to environmental stimuli, such as programmed cell death, apoptosis, protein kinase activity, and DNA binding (Fig. 3B), as well as specific GOE terms related to plant immune responses and SAR (Fig. 3C). Notably, the $\mathrm{H}_{2} \mathrm{O}_{2}$-generating PA oxidase genes PAOl (AT5G13700.1), PAO2 (AT2G43020.1), and PAO4 (AT1G65840.1) were among the genes significantly induced in response to Spm, with $P A O 1$ showing the

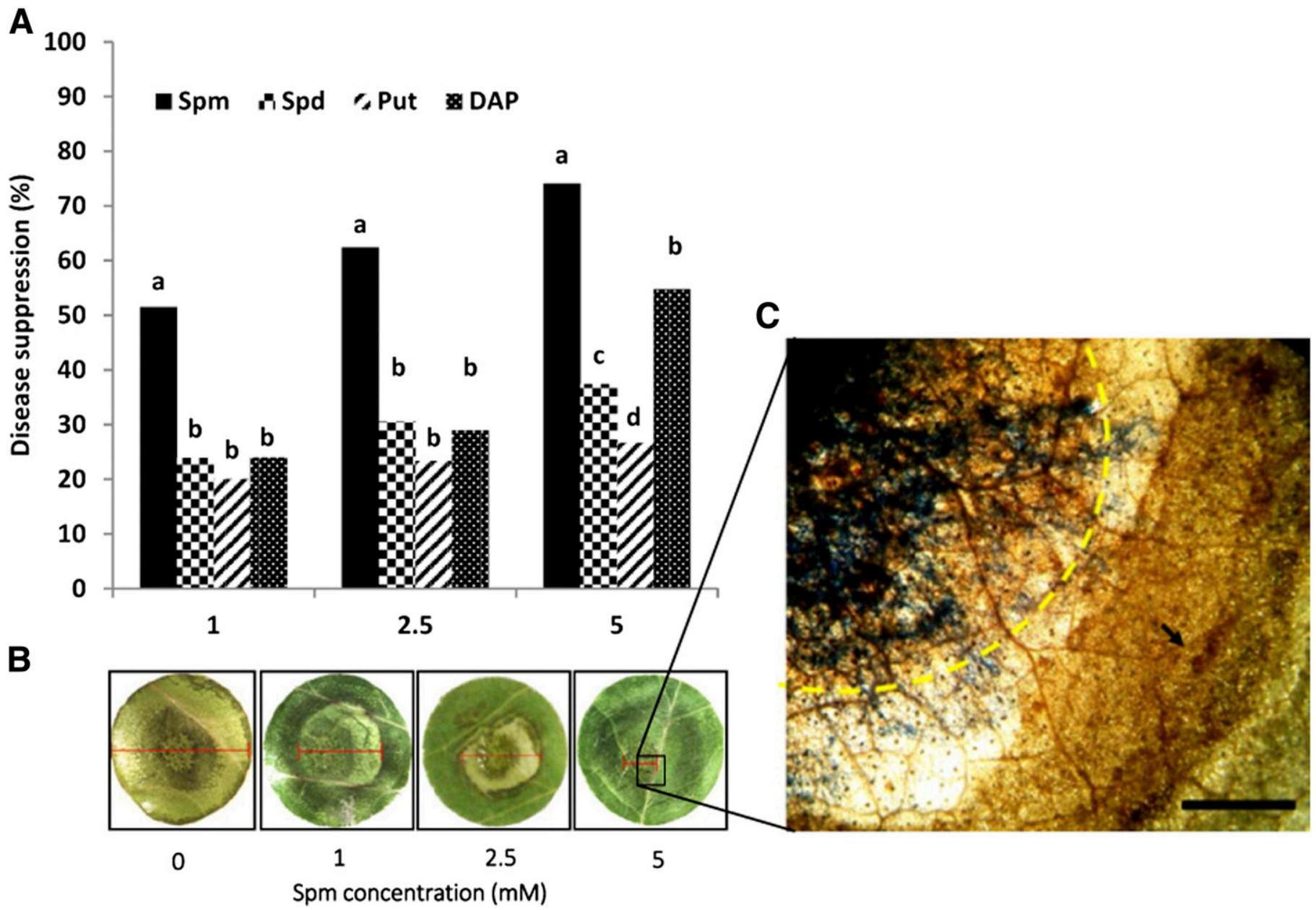

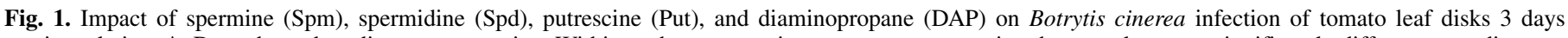

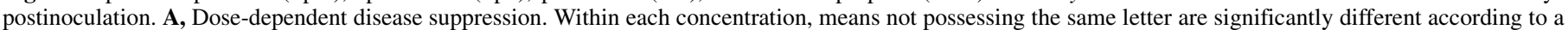

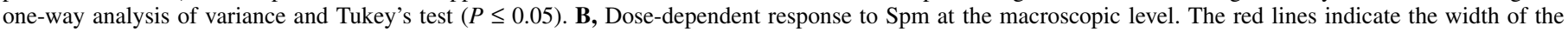

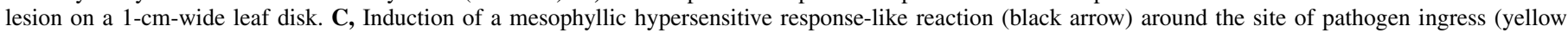

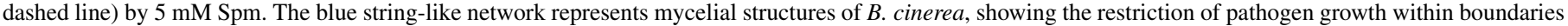
of the inoculation droplet. Scale bar $=50 \mu \mathrm{m}$. 
strongest upregulation in both SM/WM and SI/WM treatment groups (i.e., $\log _{2}$ fold change $=6.89$ and 5.96, respectively). Moreover, several key SAR-associated genes, such as flavin-dependent monooxygenase 1 (FMO1; AT1G19250.1), isochorismate synthase 1 (ICS1; AT1G74710.1) and the transcription factor WRKY70 (AT3G56400.1), were specifically identified to be strongly upregulated $\left(\log _{2}\right.$ fold change $=9.47$,
8.36, and 3.88, respectively) in response to Spm treatment (Fig. 3D and Supplementary Table S3), exhibiting distinctive expression pattern in Spm-treated samples (SM/WM and SI/WM) compared with the water-treated WI/WM group (Fig. 3E). A list of Spminduced genes involved in host immune response is provided in Supplementary Table S4.

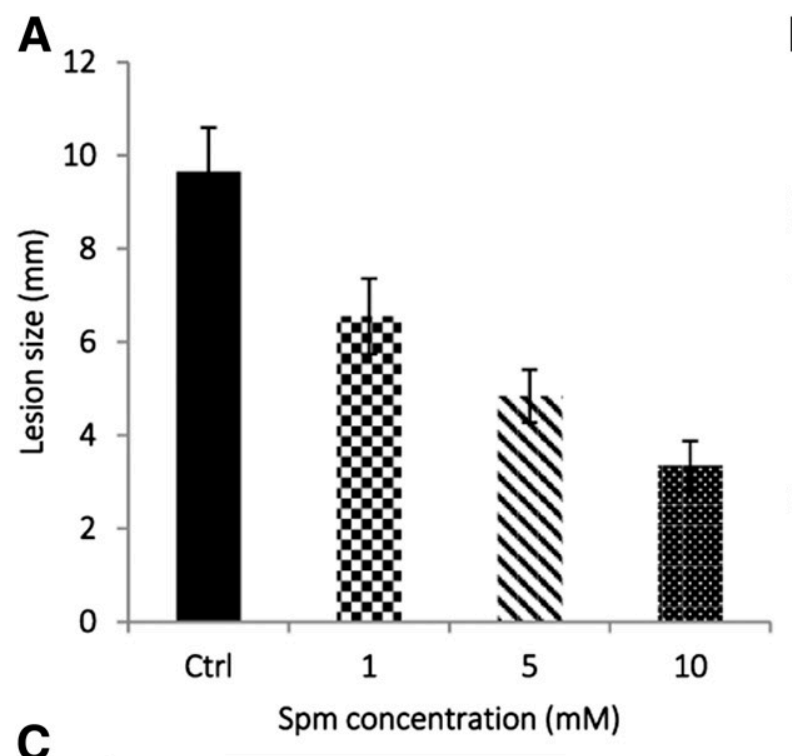

C

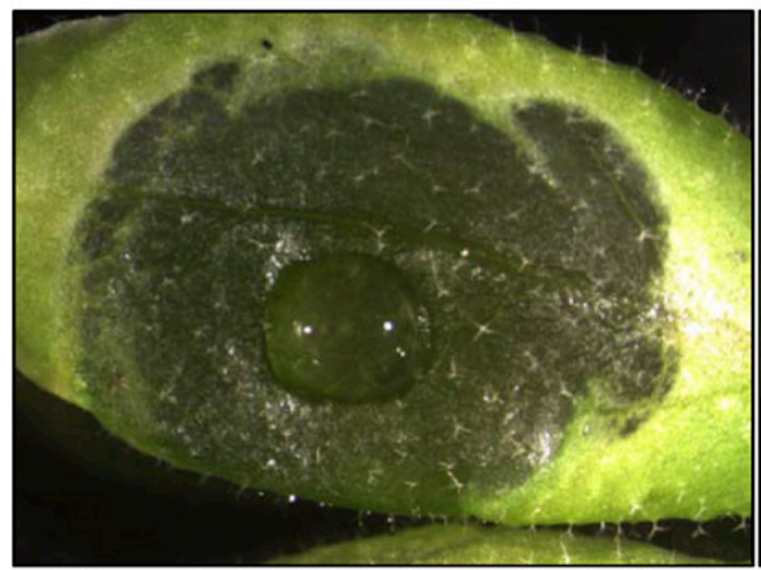

Ctrl

D

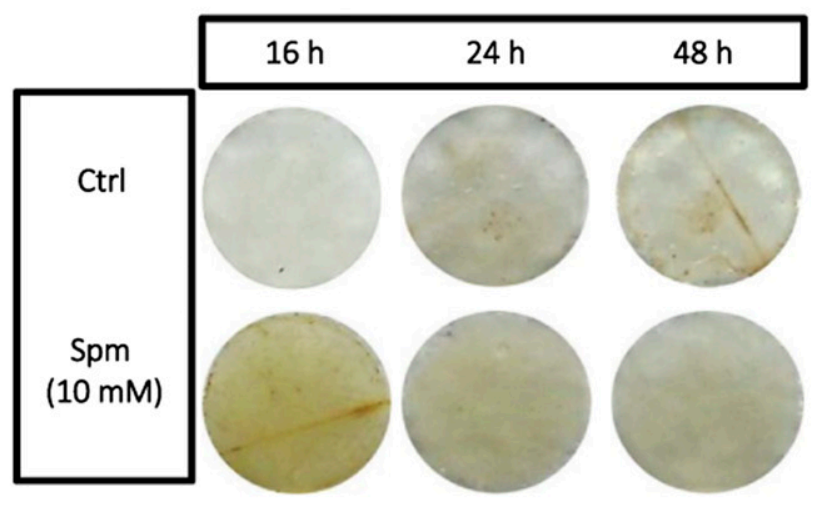

B
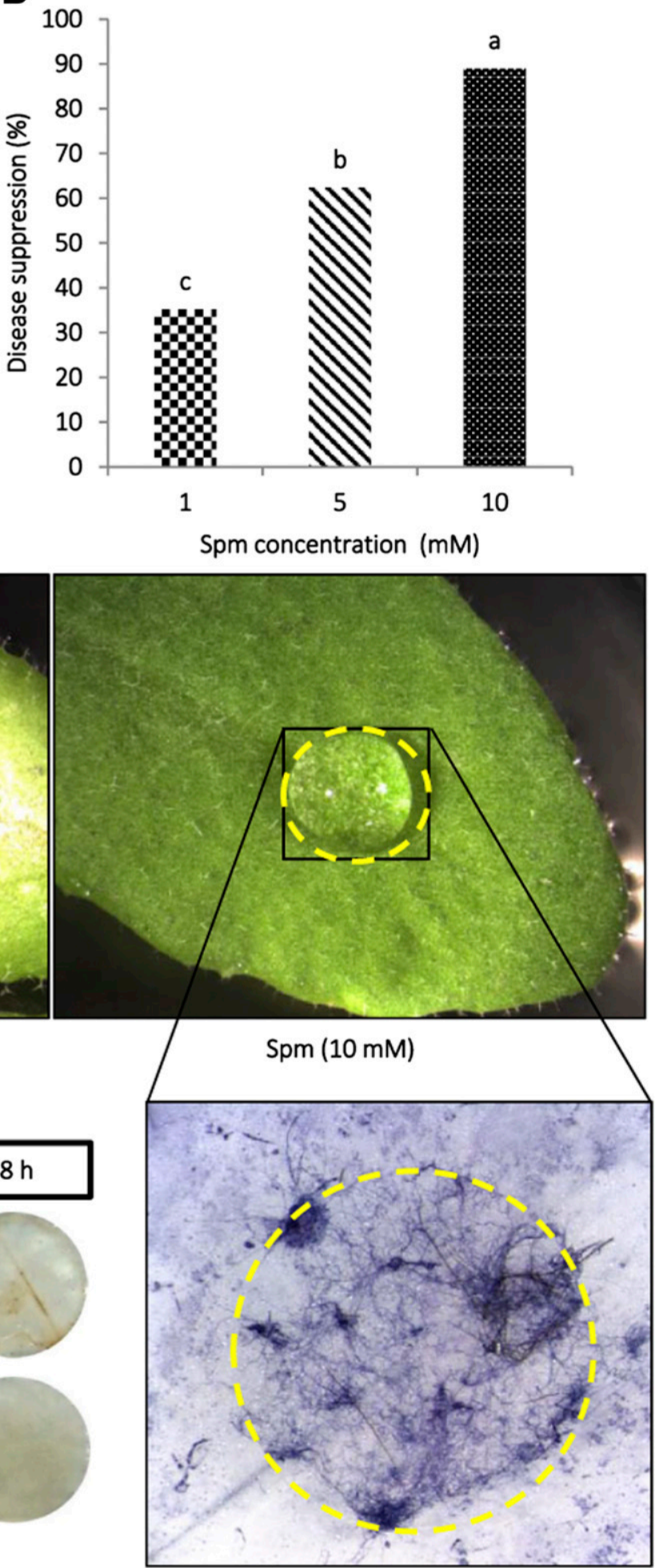

Fig. 2. Dose-dependent effect of spermine (Spm) on Botrytis cinerea infection of Arabidopsis rosette leaves 3 days postinoculation. A, Lesion size. Data represent mean \pm standard error $(n=4)$. B, Disease suppression. Bars not possessing the same letter are significantly different according to a one-way analysis of variance and Tukey's test $(P \leq 0.5)$. C, Macroscopic and microscopic symptoms after treatment with $10 \mathrm{mM}$ Spm or water (Ctrl). The micrograph (lower panel) shows normal growth of $B$. cinerea mycelia, which is fully suppressed within the boundaries of the inoculation droplet (dashed yellow line). D, Pattern of reactive oxygen species accumulation in inoculated leaf disks. 
Coapplication of Spm and SA synergistically suppressed B. cinerea infection on tomato leaf disks. Coapplication of $1 \mathrm{mM} \mathrm{SA}$ and $1 \mathrm{mM} \mathrm{Spm}$ resulted in 92, 80, and 63\% suppression of disease severity in tomato leaf disks at 3, 4, and $5 \mathrm{dpi}$, respectively (Fig. 4A and B). The level of disease suppression with the coapplication of $1 \mathrm{mM}$ Spm and SA was markedly higher than that obtained with separate applications of either Spm or SA at the corresponding time points. Notably, mycelial growth in treated samples was generally restricted to the challenged area (Fig. 4C), and both epidermal (mosaic-shaped) and mesophyllic

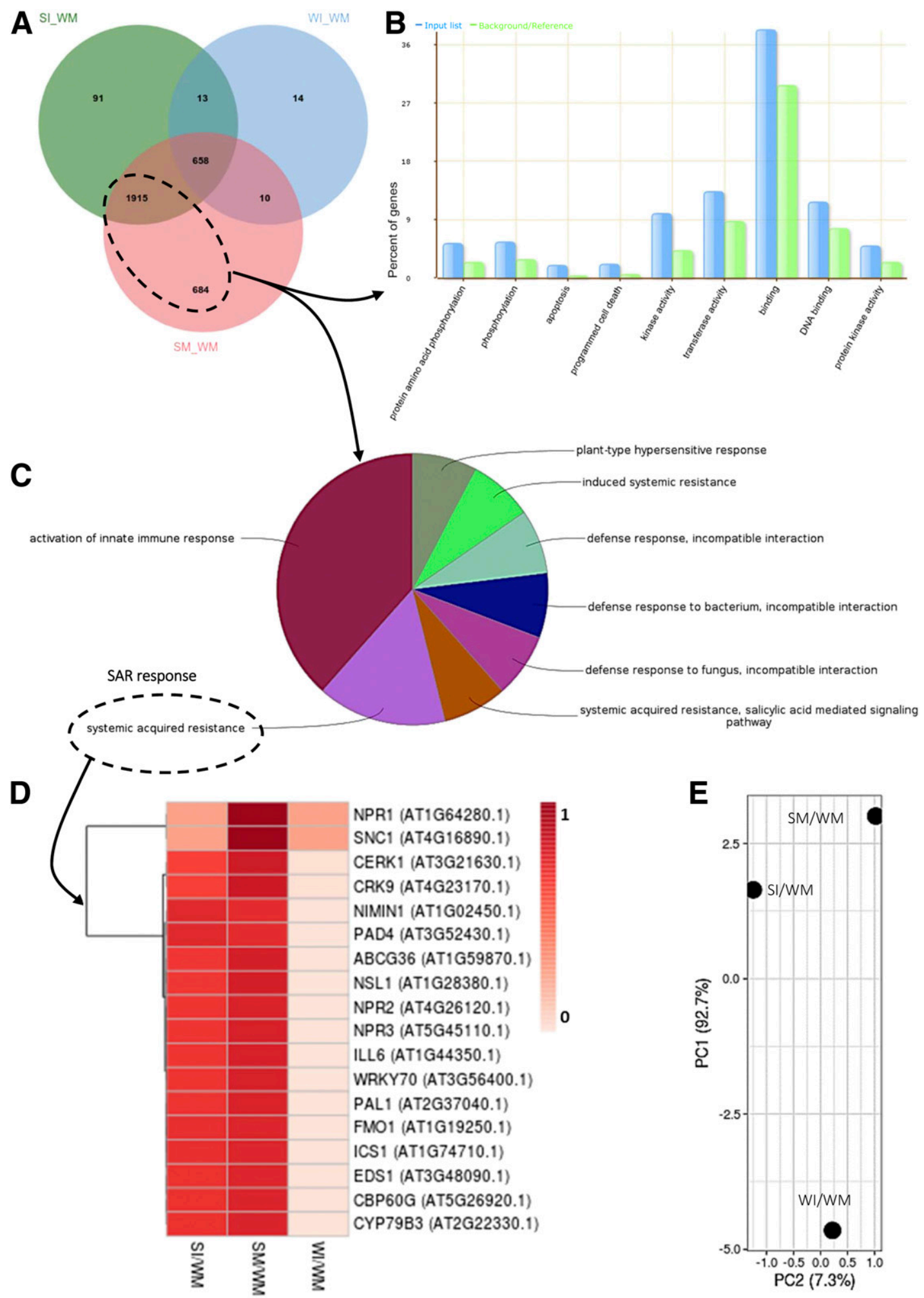

Fig. 3. Transcriptome analysis of spermine (Spm)-induced suppression of Botrytis cinerea infection on Arabidopsis rosette leaves. A, Venn diagram showing the unique and overlapping sets of genes significantly $(P \leq 0.01)$ and differentially $\left(\log _{2}\right.$ fold change $\left.\geq 2\right)$ upregulated in the various treatments (i.e., SI: spermine treated and B. cinerea inoculated; SM: spermine treated and mock inoculated; WI: water treated and B. cinerea inoculated) compared with the water control (i.e., WM: water treated and mock inoculated). The set of 2,599 differentially expressed genes (DEGs) belonging to SM-(SM $\cap$ WI; dashed area) was selected for the downstream gene ontology enrichment analysis. B and C, Enriched "Molecular Function" and "Immune Response" gene ontology terms of the selected genes. D, DEG analysis of genes involved in the induction of systemic-acquired resistance (SAR). E, Principal component (PC) analysis of the SAR-associated DEGs detected in SI/WM, SM/WM, and WI/WM. 
(cylinder-shaped) HR reactions were observed in most of the Spm/ SA-treated samples (Fig. 4D). The coapplication of $0.5 \mathrm{mM} \mathrm{Spm}$ and $3 \mathrm{mM}$ SA gave the highest synergism among the various concentration combinations tested (Fig. 4E). Furthermore, $0.5 \mathrm{mM}$
Spm did not show any inhibitory effect on in vitro growth rate and sporulation (not shown) of $B$. cinerea, whereas separate applications of $3 \mathrm{mM} \mathrm{SA}$ and a mixture of $0.5 \mathrm{mM} \mathrm{Spm}$ and $3 \mathrm{mM}$ SA had weak fungistatic effects (Fig. 5A and C). However, $3 \mathrm{mM} \mathrm{SA} \mathrm{did} \mathrm{not}$
A

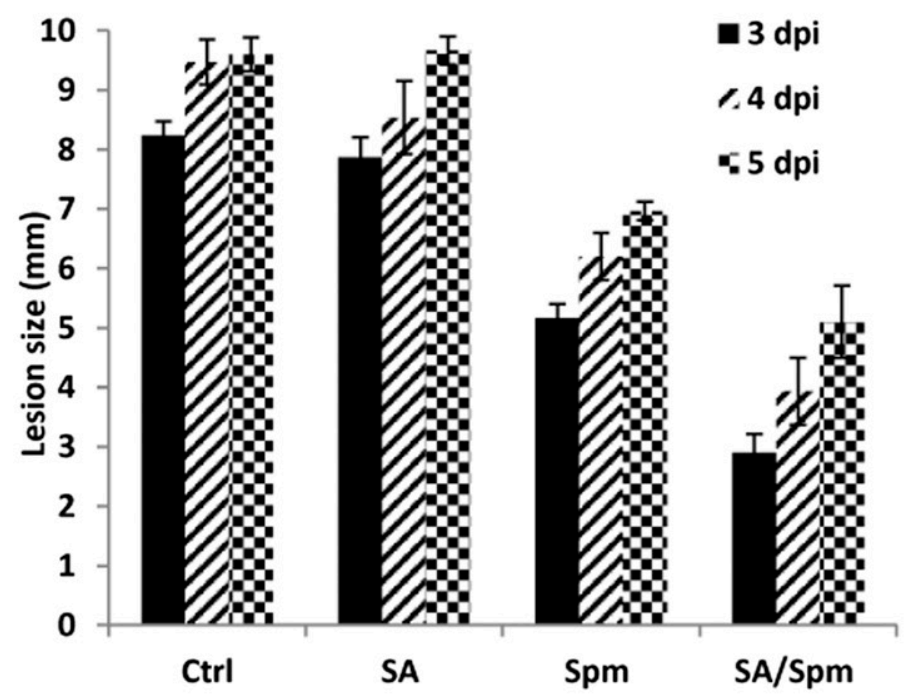

B

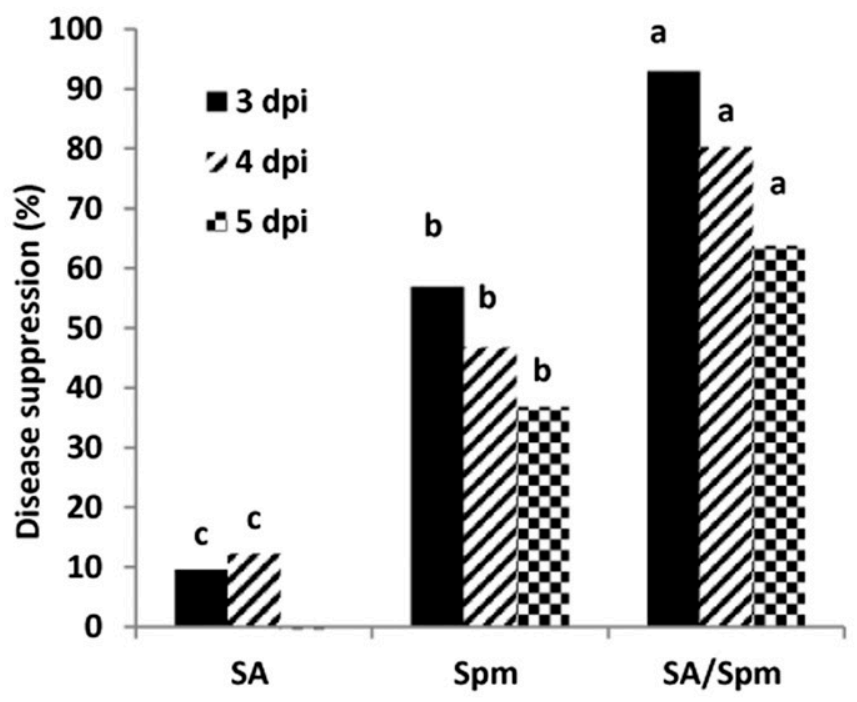

C

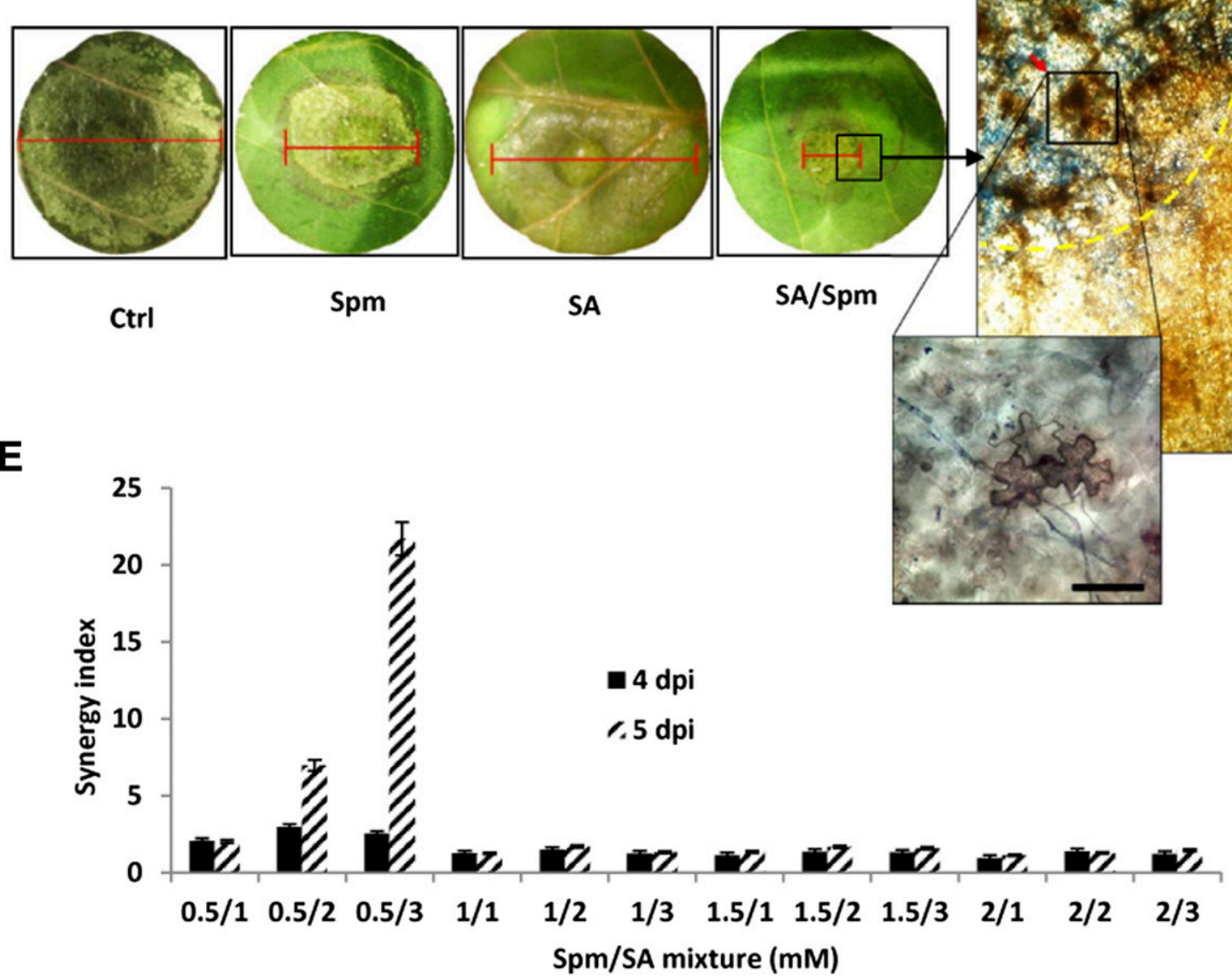

Fig. 4. Impact of coapplication of spermine (Spm) and sodium salicylate (SA), applied singly and in combination (1 mM each), on Botrytis cinerea infection on tomato leaf disks 3 to 5 days postinoculation (dpi). A, Lesion size. Data typically represent mean \pm standard error $(n=4)$. B, Disease suppression. For each dpi group, means not possessing the same letter are significantly different $(P \leq 0.05)$. C, Disease suppression 3 dpi at the macroscopic level. D, Disease suppression 3 dpi at the microscopic level. Induction of both epidermal hypersensitive response (HR; red arrow) within the challenged area (indicated by the yellow dashed line) and mesophyllic HR ring (black arrow) around the challenged area. Growth of $B$. cinerea mycelia (the blue string-like structures) is suppressed within the boundaries of the inoculation droplet. Scale bar $=50 \mu \mathrm{m}$. E, Optimization of the synergistic response for disease suppression. This is expressed as a synergy index (i.e., ratio of disease suppression with the mixture divided by the sum of the disease suppression with the single compounds). Ctrl, water. 
obviously negatively affect the growth of $B$. cinerea on tomato leaf disks (Fig. 5B). These findings indicate that the fungus responded differentially to applications or coapplications of Spm and SA in vitro and on the foliage (Fig. 5C).

Coapplication of Spm and SA potentiated early apoplastic ROS generation in tomato leaf disks inoculated with $B$. cinerea. The spatiotemporal pattern of $\mathrm{H}_{2} \mathrm{O}_{2}$ generation in tomato leaf disks revealed no accumulation in water- or SA-treated samples from 0 to 24 hpi (Fig. 6A, Ctrl and SA rows). However, strong $\mathrm{H}_{2} \mathrm{O}_{2}$ accumulation was evident within and outside the boundaries of the challenged area at 48 hpi with both treatments, which corresponded to severe lesion development by 72 hpi (Fig. 6A). Application of Spm induced moderate $\mathrm{H}_{2} \mathrm{O}_{2}$ accumulation beneath the challenged area at 8 and 12 hpi (Fig. 6A, Spm row), mainly in the apoplastic space of the leaf tissue (Fig. 6B, left panel). At 24 and $48 \mathrm{hpi}$, the Spm treatment showed $\mathrm{H}_{2} \mathrm{O}_{2}$ accumulation accompanied with dark brown HR-like spots within and slightly around the challenged area (Fig. 6A, Spm row), leading to slightly spreading lesions (Fig. 6A, Spm row) (72 hpi). A similar situation was observed at 8 and 12 hpi with the coapplication of Spm and SA (Fig. 6A, Spm/SA row). However, a restricted, highly localized accumulation of $\mathrm{H}_{2} \mathrm{O}_{2}$ within the challenged area only was evident at $48 \mathrm{hpi}$ with $\mathrm{Spm} / \mathrm{SA}$ (Fig. 6A, Spm/SA row), lending support for the strong HR and full resistance response seen at 72 hpi (Fig. 6A, $\mathrm{Spm} / \mathrm{SA}$ row). Also, Spm/SA-induced HR reactions could be detected in the epidermal layers of the challenged area at $48 \mathrm{hpi}$ (Fig. 6B, right panel). Notably, the length of $B$. cinerea hyphae on the tomato leaf surface at $8 \mathrm{hpi}$, which is the typical time for cell penetration (Asselbergh et al. 2007), did not differ between the water- and Spm/SA-treated samples (Fig. 6C and D), suggesting that $\mathrm{Spm}$ and SA coapplication did not adversely affect $B$. cinerea growth.

Coapplication of Spm and SA suppressed B. cinerea infection on both sprayed and systemic (nonsprayed) detached leaves. Spraying of Spm and SA mixtures $(1 / 3 \mathrm{mM})$ onto intact leaves of tomato plants $16 \mathrm{~h}$ before inoculation
A

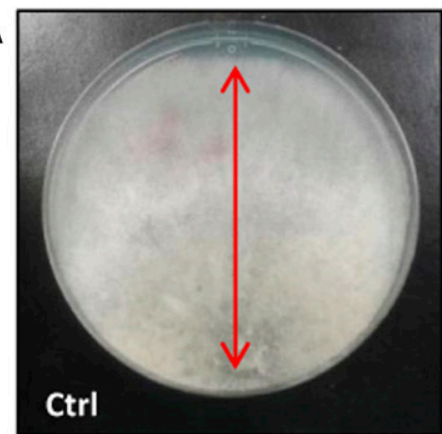

B

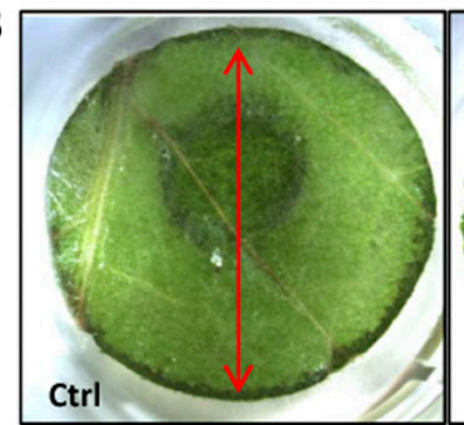

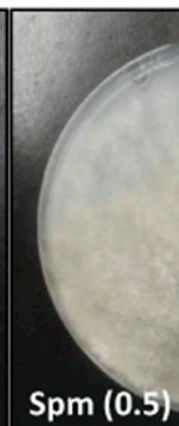

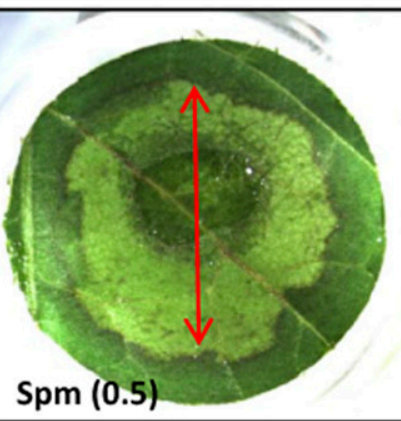

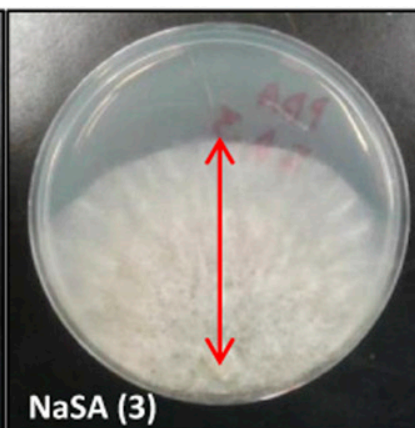

NaSA (3)

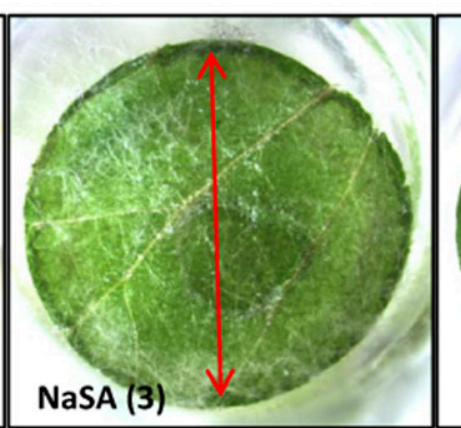

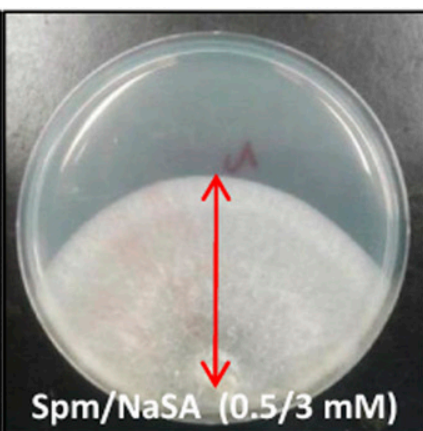

Spm/NaSA $(0.5 / 3 \mathrm{mM})$

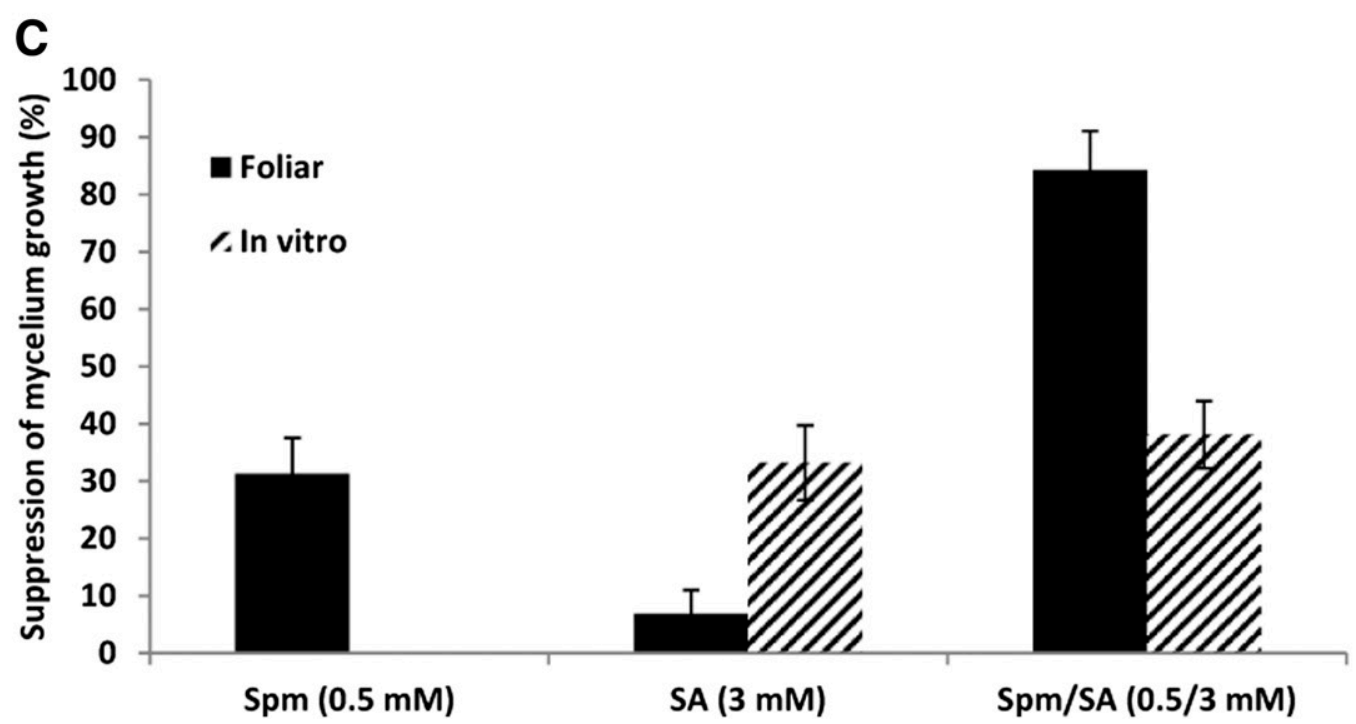

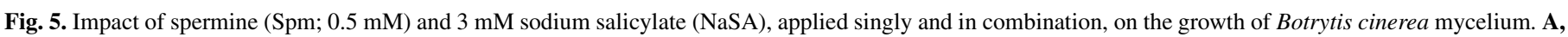

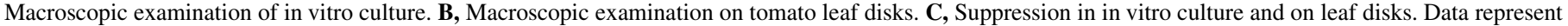
the mean \pm standard error of three biological replicates. Ctrl, water. 
suppressed disease severity in the sprayed leaves by $41 \%$ at $3 \mathrm{dpi}$ (Fig. 7A to C). Similarly, when only the first, second, and third leaves of intact plants were sprayed with the same Spm/SA mixture, $40 \%$ disease suppression was observed in the upper systemic nonsprayed leaves (the fifth and sixth leaves) at $3 \mathrm{dpi}$ (Fig. 7D to F).

\section{DISCUSSION}

In this study, we report that, among the major PAs in plants (i.e., Spm, Spd, and Put) and their catabolic product DAP, the exogenous application of Spm had the greatest potential to induce effective control of $B$. cinerea infection in tomato, bean, and the

A

Ctrl

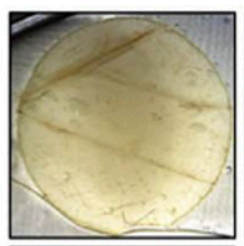

SA
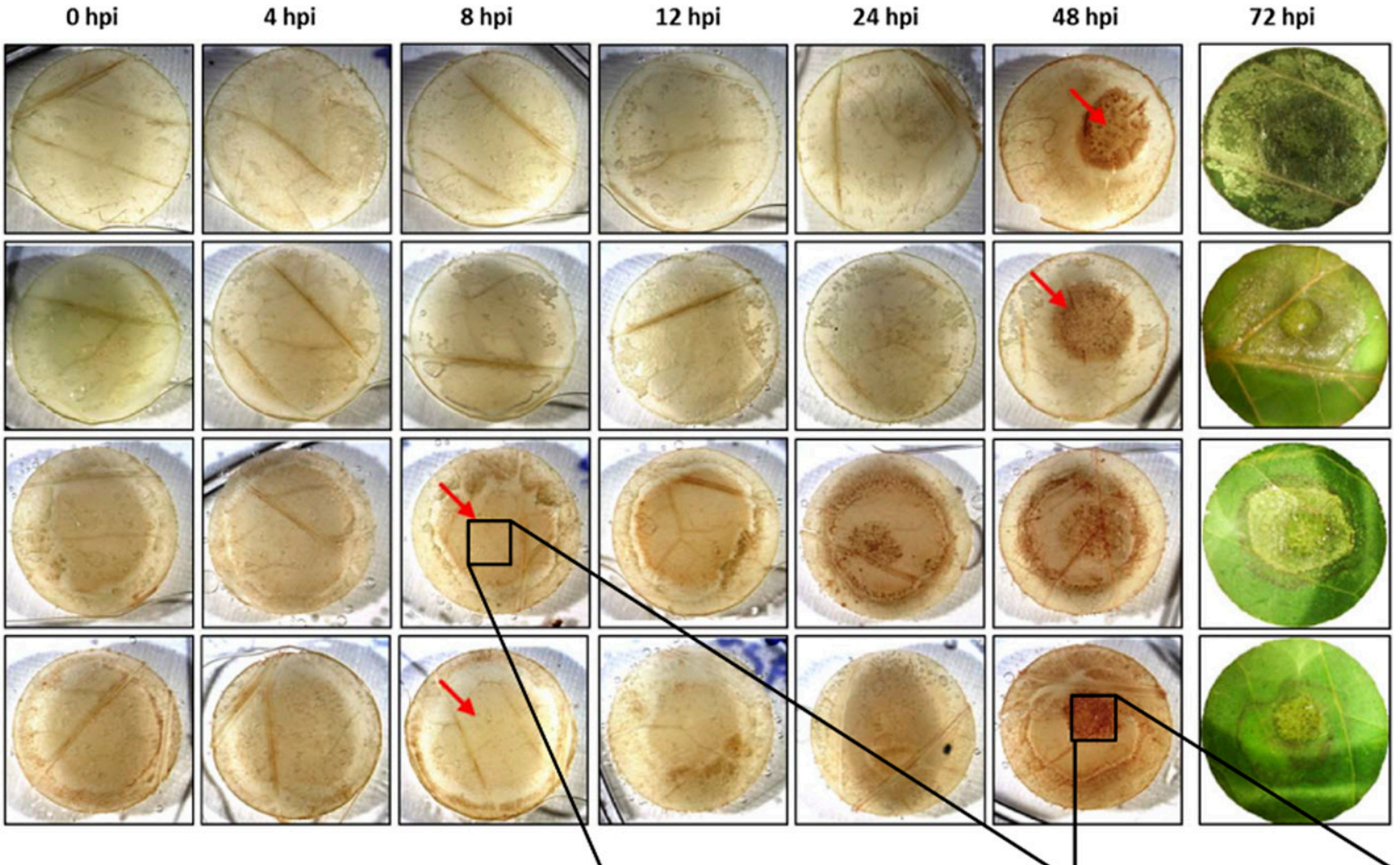

Spm-SA
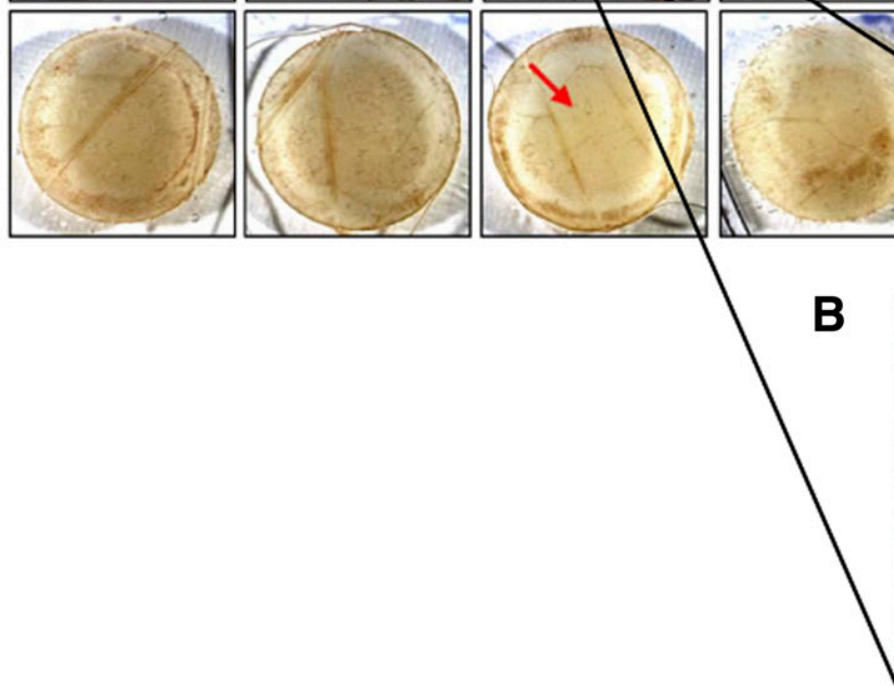

C

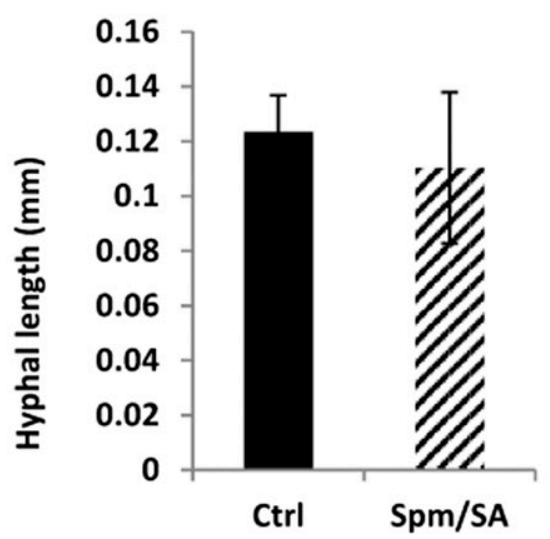

D

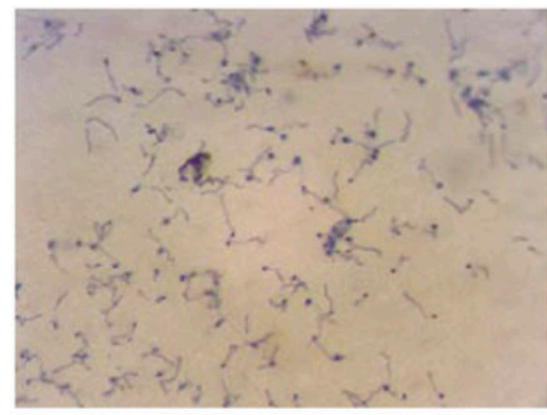

Ctrl

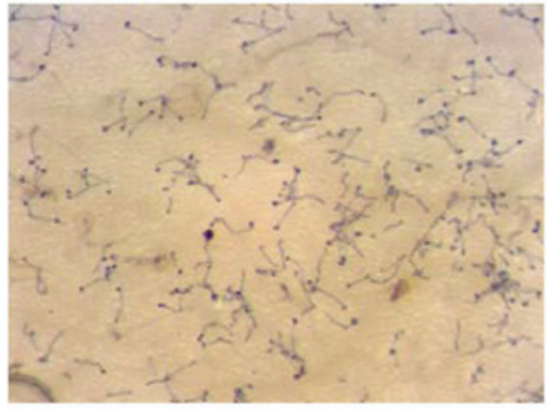

$\mathrm{Spm} / \mathrm{SA}$

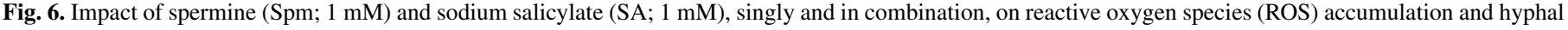

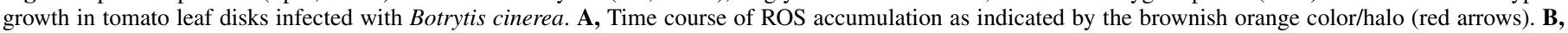

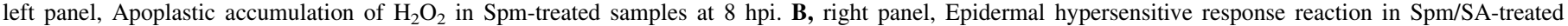
samples at $72 \mathrm{~h}$ postinoculation (hpi). Scale bar $=50 \mu \mathrm{m}$. C and D, Hyphae growth at 8 hpi on water (Ctrl)- and Spm/SA-treated tomato leaf disks. 
genus Arabidopsis and seemed to activate potent plant defense mechanism(s) rather than exert an antimicrobial effect. This was confirmed through RNA-seq-based transcriptome analysis showing that exogenous application of Spm induced immune response and SAR-associated genes in the genus Arabidopsis. Moreover, coapplication of Spm and SA was found to induce even stronger protection than Spm alone in a synergistic manner in tomato plants. Additionally, the Spm/SA mixture induced resistance against $B$. cinerea in nontreated leaves detached from the same plants, suggesting that it acts in a systemic manner.

Our results suggest a special role for Spm in modulating plant defense response against $B$. cinerea infection. Previously, Yamakawa et al. (1998) reported that exogenous application of Spm reduces susceptibility of tobacco leaves to tomato mosaic virus in a dose-dependent manner. Of all of the PAs tested, including Put and Spd, only Spm induces strong upregulation of the classic defense marker gene $P R 1$ through an SA-independent pathway. Similarly, exogenous Spm fully suppresses cauliflower mosaic virus yellow in the genus Arabidopsis, whereas Spd and Put have little and no effect, respectively (Mitsuya et al. 2009). Other research indicated that Spm treatment strongly induces different defense-related genes in Arabidopsis seedlings, whereas similar doses of Put and Spd treatments are totally ineffective, and higher levels of Spm in Spm synthase overexpression lines of the genus Arabidopsis are causally linked to higher tolerance to the bacterial pathogen Pseudomonas syringae and the oomycete $H$. arabidopsidis (Marco et al. 2014). Likewise, our transcriptome analysis indicated that Spm induced several defense-related genes in the genus Arabidopsis, particularly those involved with the establishment of SAR (Fig. 3C and D). FMO1, ICS1, and WRKY70 in particular were strongly upregulated. Previous research has shown that Arabidopsis fmo mutants are impaired in the establishment of SAR triggered by both avirulent and virulent Pseudomonas syringae bacteria as well as the systemic accumulation of SA and systemic expression of several defense-related genes (Mishina and Zeier 2006). Furthermore, ICS1 is essential for the biosynthesis of SAR-associated SA (Wildermuth et al. 2001), and WRKY70 integrates signals from salicylate- and jasmonatemediated defense pathways, which are mutually antagonistic ( $\mathrm{Li}$ et al. 2004). Notably, WRKY70 is involved in the regulation of SA biosynthesis and the SAR response (Wang et al. 2006). Additional research is clearly necessary to unravel molecular mechanisms that control Spm-induced SAR.

Although the exact function of the PAs, particularly Spm, during incompatible plant-pathogen interactions is not yet known, the favored hypothesis attributes the induction of cell death and HR and
A

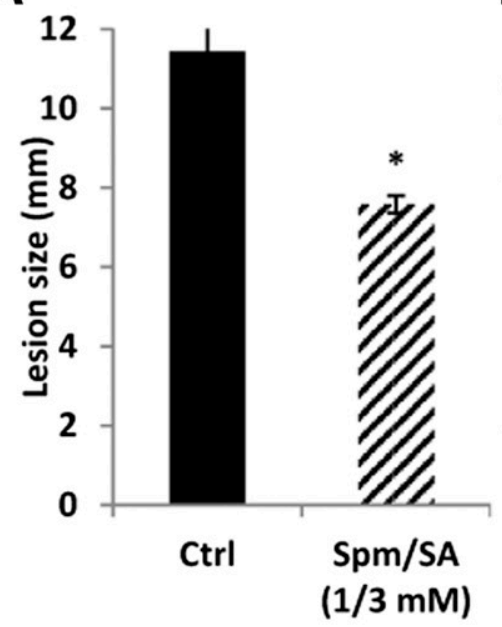

B

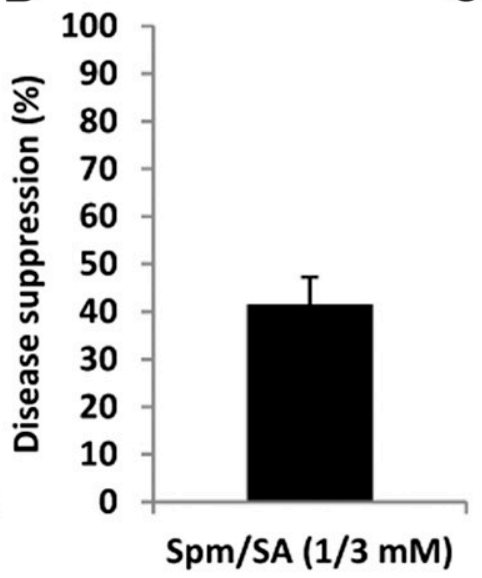

C
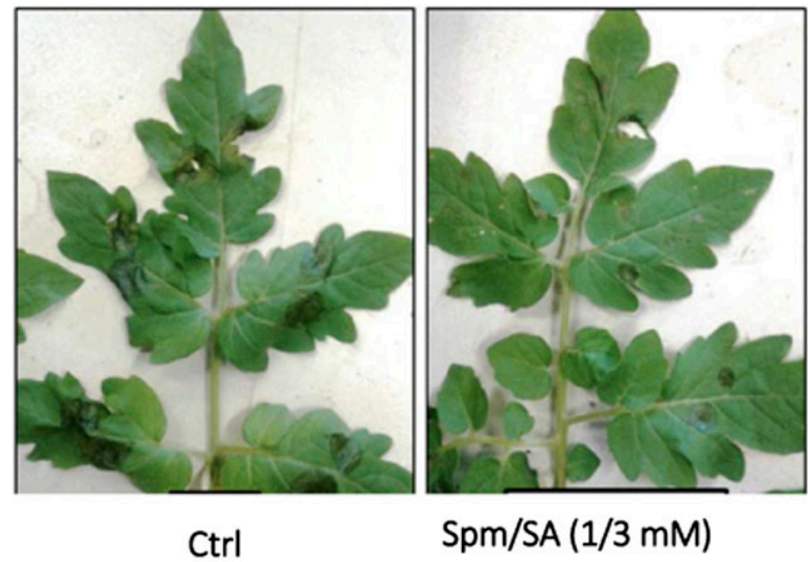

F

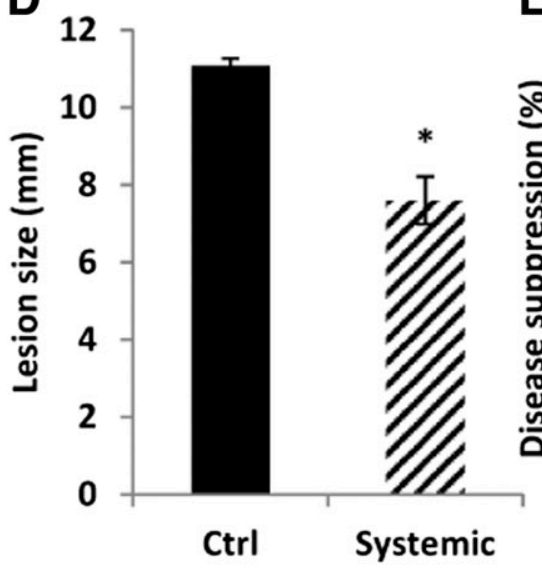

E

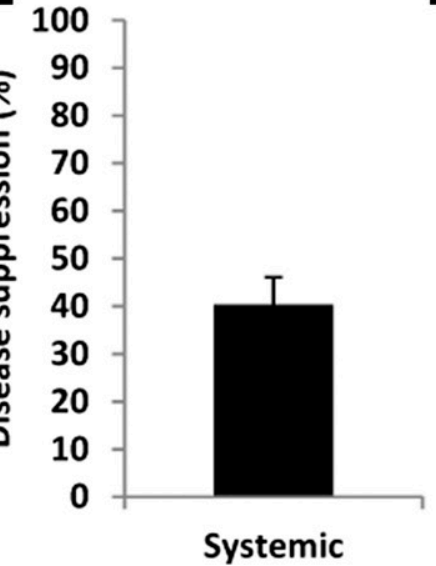

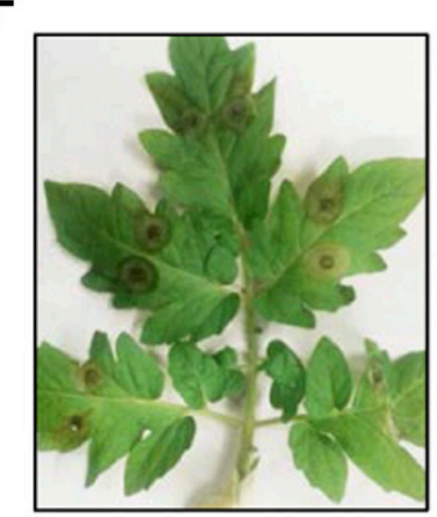

Ctrl

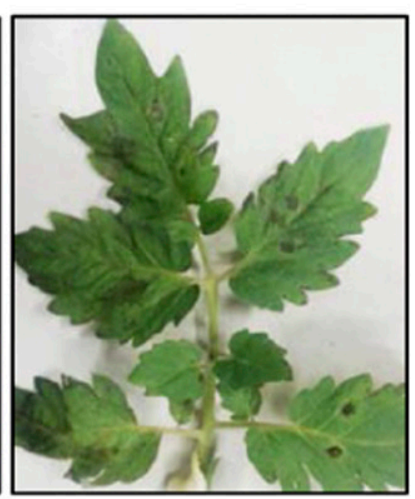

Systemic

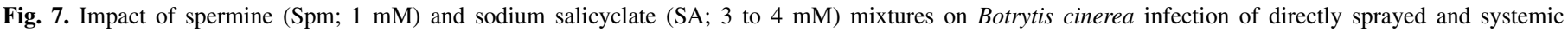

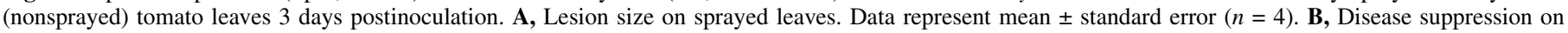

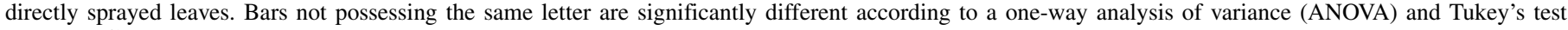

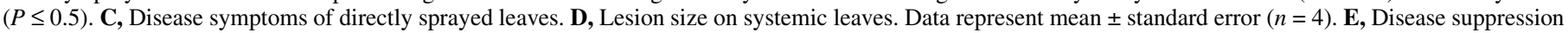

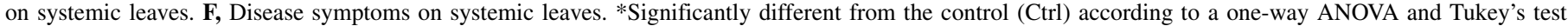
$(P \leq 0.5)$. 
consequently, the expression of defense genes to $\mathrm{H}_{2} \mathrm{O}_{2}$ production via PA oxidation (Jiménez-Bremont et al. 2014; Seifi et al. 2013b; Takahashi et al. 2004; Walters 2003a). In this study, we found traces of early apoplastic $\mathrm{H}_{2} \mathrm{O}_{2}$ accumulation in Spm- and Spm/ SA-treated leaves, which did not coincide with the signs of epidermal or mesophyllic HR at 4 and 8 hpi (Fig. 6), suggesting that the level of the early $\mathrm{H}_{2} \mathrm{O}_{2}$ burst was tightly controlled to prevent cell death. A similar situation was observed in Spmtreated Arabidopsis leaves (Fig. 2D), and it was associated with the induction of the $\mathrm{H}_{2} \mathrm{O}_{2}$-generating PAOl (AT5G13700.1), PAO2 (AT2G43020.1), and PAO4 (AT1G65840.1) genes at $16 \mathrm{hpi}$. Such early $\mathrm{H}_{2} \mathrm{O}_{2}$ generation impulses in response to pathogen ingress are generally known to serve a defense-associated signaling role, activating downstream defense mechanisms in the host tissue (Conrath et al. 2006). Notably, at later stages of the interaction (i.e., 24 and 48 hpi), clear signs of strong but highly localized HR reaction were detected in tomato leaf disks (Fig. 6). Presumably, restricting the extent of oxidative stress during early stages of the interaction followed by a highly localized HR prevents the cell death-favoring invasion strategy of the necrotrophic $B$. cinerea from being initiated on penetration of the host tissue. This finding is in accordance with previous studies showing that, despite early $\mathrm{H}_{2} \mathrm{O}_{2}$ generation in the sitiens tomato mutant, the extent of cell death is tightly controlled, leading to strong HR-dependent resistance against $B$. cinerea (Asselbergh et al. 2007; Curvers et al. 2010; Seifi et al. 2013a). Interestingly, the SA signaling pathway plays an important role in the sitiens resistance response to B. cinerea (Audenaert et al. 2002). Moreover, exogenous application of the SA analog, benzothiadiazole, reduces susceptibility to $B$. cinerea in tomato, bean, and cucumber (Azami-Sardooei et al. 2013). These observations may explain why coapplication of Spm and SA in tomato led to a synergistically higher level of resistance to B. cinerea (Fig. 2), presumably through the induction of a stronger SAR response in the host. An interaction between Spm and SA has been reported for the chickpea-Fusarium oxysporum pathosystem, where coapplication of Spm and SA elevates the activities of two defense-related enzymes, polyphenol oxidase and phenylalanine ammonia lyase (Raju et al. 2008). Notably, a wilt diseaseresistant cultivar exhibits an additive interaction between Spm $(0.01 \mathrm{mM})$ and SA $(0.8 \mathrm{mM})$, whereas a susceptible cultivar does not (Raju et al. 2009). Likewise, the Spm and SA coapplication induces defense-associated protease inhibitors in the resistant cultivar only (Raju et al. 2009). In our study, however, the susceptible wild-type tomato was highly responsive to Spm and the Spm/SA mixture, resulting in strong resistance against B. cinerea. Collectively, our data highlight the importance of an SA-natured SAR response in the induction of resistance to $B$. cinerea and do not support the widely held view that SA-mediated defense pathways are specifically associated with immunity against biotrophic pathogens, whereas jasmonatedependent defense responses are effective only against necrotrophs (Glazebrook 2005).

The economic importance of gray mold disease (Dean et al. 2012) highlights the necessity for effective but environmentally safe controls against $B$. cinerea. To our knowledge, this study is the first report of effective gray mold control on tomato, bean, and the genus Arabidopsis by exogenous application of the PA Spm as well as the synergistic interaction of Spm and SA coapplication on induced resistance in tomato. Considering the natural origin of the two compounds, this finding could be a significant step toward establishing novel ecofriendly methods to control not only Botrytis blight but possibly, many other important plant pathogens with a similar necrotrophic mode of infection, such as Sclerotinia sclerotiorum and Alternaria solani. Furthermore, our transcriptomics data provided a baseline for future mechanistic studies to further elucidate the molecular basis underlying Spm-induced defense activation in plants.

\section{ACKNOWLEDGMENTS}

We thank Kelly Tanaka and Christopher Trobacher for their support and helpful discussions. We also thank Alireza Navabi for providing facilities for bioinformatics analyses.

\section{LITERATURE CITED}

Alcázar, R., Marco, F., Cuevas, J. C., Patron, M., Ferrando, A., Carrasco, P., Tiburcio, A. F., and Altabella, T. 2006. Involvement of polyamines in plant response to abiotic stress. Biotechnol. Lett. 28:1867-1876.

Andrews, S. 2010. FastQC A Quality Control Tool for High Throughput Sequence Data. https://www.bioinformatics.babraham.ac.uk/projects/fastqc/

Aranega-Bou, P., de la O Leyva, M., Finiti, I., García-Agustín, P., and González-Bosch, C. 2014. Priming of plant resistance by natural compounds. Hexanoic acid as a model. Front. Plant Sci. 5:488.

Asselbergh, B., Curvers, K., Franca, S. C., Audenaert, K., Vuylsteke, M., Van Breusegem, F., and Höfte, M. 2007. Resistance to Botrytis cinerea in sitiens, an abscisic acid-deficient tomato mutant, involves timely production of hydrogen peroxide and cell wall modifications in the epidermis. Plant Physiol. 144:1863-1877.

Audenaert, K., De Meyer, G. B., and Hofte, M. M. 2002. Abscisic acid determines basal susceptibility of tomato to Botrytis cinerea and suppresses salicylic acid-dependent signaling mechanisms. Plant Physiol. 128:491-501.

Azami-Sardooei, Z., Seifi, H. S., De Vleesschauwer, D., and Höfte, M. 2013. Benzothiadiazole (BTH)-induced resistance against Botrytis cinerea is inversely correlated with vegetative and generative growth in bean and cucumber, but not in tomato. Australas. Plant Pathol. 42:485-490.

Bardou, P., Mariette, J., Escudié, F., Djemiel, C., and Klopp, C. 2014. jvenn: An interactive Venn diagram viewer. BMC Bioinformatics 15:293.

Bindea, G., Mlecnik, B., Hackl, H., Charoentong, P., Tosolini, M., Kirilovsky, A., Fridman, W.-H., Pagès, F., Trajanoski, Z., and Galon, J. 2009. ClueGO: A Cytoscape plug-in to decipher functionally grouped gene ontology and pathway annotation networks. Bioinformatics 25:1091-1093.

Bolger, A. M., Lohse, M., and Usadel, B. 2014. Trimmomatic: A flexible trimmer for Illumina sequence data. Bioinformatics 30:2114-2120.

Cona, A., Rea, G., Angelini, R., Federico, R., and Tavladoraki, P. 2006. Functions of amine oxidases in plant development and defence. Trends Plant Sci. 11:80-88.

Conrath, U., Beckers, G. J. M., Flors, V., García-Agustín, P., Jakab, G., Mauch, F., Newman, M.-A., Pieterse, C. M. J., Poinssot, B., Pozo, M. J., Pugin, A., Schaffrath, U., Ton, J., Wendehenne, D., Zimmerli, L., and Mauch-Mani, B. 2006. Priming: Getting ready for battle. Mol. Plant-Microbe Interact. 19: 1062-1071.

Curvers, K., Seifi, H., Mouille, G., de Rycke, R., Asselbergh, B., Van Hecke, A., Vanderschaeghe, D., Höfte, H., Callewaert, N., Van Breusegem, F., and Höfte, M. 2010. Abscisic acid deficiency causes changes in cuticle permeability and pectin composition that influence tomato resistance to $\mathrm{Bo}$ trytis cinerea. Plant Physiol. 154:847-860.

Dean, R., Van Kan, J. A. L., Pretorius, Z. A., Hammond-Kosack, K. E., Di Pietro, A., Spanu, P. D., Rudd, J. J., Dickman, M., Kahmann, R., Ellis, J., and Foster, G. D. 2012. The top 10 fungal pathogens in molecular plant pathology. Mol. Plant Pathol. 13:414-430.

Gaffney, T., Friedrich, L., Vernooij, B., Negrotto, D., Nye, G., Uknes, S., Ward, E., Kessmann, H., and Ryals, J. 1993. Requirement of salicylic acid for the induction of systemic acquired resistance. Science 261:754-756.

Galston, A. W., and Sawhney, R. K. 1990. Polyamines in plant physiology. Plant Physiol. 94:406-410.

Glazebrook, J. 2005. Contrasting mechanisms of defense against biotrophic and necrotrophic pathogens. Annu. Rev. Phytopathol. 43:205-227.

Heath, M. C. 2000. Nonhost resistance and nonspecific plant defenses. Curr. Opin. Plant Biol. 3:315-319.

Jiménez-Bremont, J. F., Marina, M., Guerrero-González, M. de la L., Rossi, F. R., Sánchez-Rangel, D., Rodríguez-Kessler, M., Ruiz, O. A., and Gárriz, A. 2014. Physiological and molecular implications of plant polyamine metabolism during biotic interactions. Front. Plant Sci. 5:95.

Jones, J. D. G., and Dangl, J. L. 2006. The plant immune system. Nature 444: 323-329.

Jurick, W. M., II, Macarisin, O., Gaskins, V. L., Park, E., Yu, J., Janisiewicz, W., and Peter, K. A. 2017. Characterization of postharvest fungicideresistant Botrytis cinerea isolates from commercially stored apple fruit. Phytopathology 107:362-368.

Li, J., Brader, G., and Palva, E. T. 2004. The WRKY70 transcription factor: A node of convergence for jasmonate-mediated and salicylate-mediated signals in plant defense. Plant Cell 16:319-331.

Marco, F., Busó, E., and Carrasco, P. 2014. Overexpression of SAMDC1 gene in Arabidopsis thaliana increases expression of defense-related genes as well as resistance to Pseudomonas syringae and Hyaloperonospora arabidopsidis. Front. Plant Sci. 5:115. 
Meldau, S., Ullman-Zeunert, L., Govind, G., Bartram, S., and Baldwin, I. T. 2012. MAPK-dependent JA and SA signalling in Nicotiana attenuata affects plant growth and fitness during competition with conspecifics. BMC Plant Biol. 12:213.

Minocha, R., Majumdar, R., and Minocha, S. C. 2014. Polyamines and abiotic stress in plants: A complex relationship. Front. Plant Sci. 5:175.

Mishina, T. E., and Zeier, J. 2006. The Arabidopsis flavin-dependent monooxygenase FMO1 is an essential component of biologically induced systemic acquired resistance. Plant Physiol. 141:1666-1675.

Mitsuya, Y., Takahashi, Y., Berberich, T., Miyazaki, A., Matsumura, H., Takahashi, H., Terauchi, R., and Kusano, T. 2009. Spermine signaling plays a significant role in the defense response of Arabidopsis thaliana to cucumber mosaic virus. J. Plant Physiol. 166:626-643.

Raju, S., Jayalakshmi, S., and Sreeramulu, K. 2008. Comparative study on the induction of defense related enzymes in two different cultivars of chickpea (Cicer arietinum L.) genotypes by salicylic acid, spermine and Fusarium oxysporum f. sp. ciceri. Aust. J. Crop Sci. 2:121-140.

Raju, S., Jayalakshmi, S. K., and Sreeramulu, K. 2009. Differential elicitation of proteases and protease inhibitors in two different genotypes of chickpea (Cicer arietinum) by salicylic acid and spermine. J. Plant Physiol. 166: 1015-1022.

Seifi, H. S., Curvers, K., De Vleesschauwer, D., Delaere, I., Aziz, A., and Höfte, M. 2013a. Concurrent overactivation of the cytosolic glutamine synthetase and the GABA shunt in the ABA-deficient sitiens mutant of tomato leads to resistance against Botrytis cinerea. New Phytol. 199: 490-504.

Seifi, H. S., Van Bockhaven, J., Angenon, G., and Höfte, M. 2013b. Glutamate metabolism in plant disease and defense: Friend or foe? Mol. Plant-Microbe Interact. 26:475-485.

Takahashi, Y., Berberich, T., Miyazaki, A., Seo, S., Ohashi, Y., and Kusano, T. 2003. Spermine signalling in tobacco: Activation of mitogen-activated protein kinases by spermine is mediated through mitochondrial dysfunction. Plant J. 36:820-829.
Takahashi, Y., Uehara, Y., Berberich, T., Ito, A., Saitoh, H., Miyazaki, A., Terauchi, R., and Kusano, T. 2004. A subset of hypersensitive response marker genes, including $H S R 203 J$, is the downstream target of a spermine signal transduction pathway in tobacco. Plant J. 40:586-595.

Tian, T., Liu, Y., Yan, H., You, Q., Yi, X., Du, Z., Xu, W., and Su, Z. 2017. agriGO v2.0: A GO analysis toolkit for the agricultural community, 2017 update. Nucleic Acids Res. 45:W122-W129.

Trapnell, C., Roberts, A., Goff, L., Pertea, G., Kim, D., Kelley, D. R., Pimentel, H., Salzberg, S., Rinn, J., and Pachter, L. 2012. Differential gene and transcript expression analysis of RNA-seq experiments with TopHat and Cufflinks. Nat. Protoc. 7:562-578.

Walters, D. 2003a. Polyamines and plant disease. Phytochemistry 64:97-107.

Walters, D. 2003b. Resistance to plant pathogens: Possible roles for free polyamines and polyamine catabolism. New Phytol. 159:109-115.

Walters, D., Walsh, D., Newton, A., and Lyon, G. 2005. Induced resistance for plant disease control: Maximizing the efficacy of resistance elicitors. Phytopathology 95:1368-1373.

Wang, D., Amornsiripanitch, N., and Dong, X. 2006. A genomic approach to identify regulatory nodes in the transcriptional network of systemic acquired resistance in plants. PLoS Pathol. 2:e123.

Wildermuth, M. C., Dewdney, J., Wu, G., and Ausubel, F. M. 2001. Isochorismate synthase is required to synthesize salicylic acid for plant defence. Nature 414:562-565.

Williamson, B., Tudzynski, B., Tudzynski, P., and Van Kal, J. A. L. 2007. Botrytis cinerea: The cause of grey mould disease. Mol. Plant Pathol. 8:561-580.

Yamakawa, H., Kamada, H., Satoh, M., and Ohashi, Y. 1998. Spermine is a salicylate-independent endogenous inducer for both tobacco acidic pathogenesis-related proteins and resistance against tobacco mosaic virus infection. Plant Physiol. 118:1213-1222.

Zhao, M., Zhou, Y. J., Dong Li, Z., Wei Song, W., Jiu Tan, Y., and Tan, H. 2009. Boty-II, a novel LTR retrotransposon in Botrytis cinerea B05.10 revealed by genomic sequence. Electron. J. Biotechnol. http://www.ejbiotechnology. info/content/vol12/issue3/full/5/index.html 